\title{
Design for Sustainability and Reconfigurable Manufacturing Systems - An Critical Analysis
}

\author{
${ }^{1}$ Francisco Pedro \\ ${ }^{1}$ University of Minho, Braga, Portugal \\ ${ }^{1}$ franciscobraga1211@gmail.com
}

\begin{abstract}
Article Info
Journal of Machine and Computing (http://anapub.co.ke/journals/jmc/jmc.html)

Doi : https://doi.org/10.53759/7669/jmc202101002

Received 12 October 2020; Revised form 20 November 2020; Accepted 25 December 2020;

Available online 05 January 2021.

(C)2021 The Authors. Published by AnaPub Publications.

This is an open access article under the CC BY-NC-ND license. (http://creativecommons.org/licenses/by-nc-nd/4.0/)

Abstract - Manufacturing Systems (MS) are a fundamental means of establishing profitable engineering operations in mechanical firms. The technical and physical structures of MS determine the dimension of adaption and signify the measure of freedom of engineers of MS. This contribution discusses the Reconfiguring Cellular Manufacturing System (RCMS) concept, which considers the design of systems as cyclical activities whereby the makers of decisions can determine the MS's system capacities and capabilities. The standard methodologies to support the design of MS are optimization and analysis of MS. The timeframe essential for evaluating numerous designs is a fundamental factor, which can also discourage the application of these systems. Resultantly, there is a threat of missed assessment of suitable designs when the number and quality of the solution users being assessed is based on the users' intuition with respect to the potential design result.
\end{abstract}

Keyword - Manufacturing Systems (MS), Reconfiguring Cellular Manufacturing System (RCMS), Computational Design Synthesis (CDS), Flexible Manufacturing Systems (FMS)

\section{INTRODUCTION}

In the automated designing of engineered tasks, the Computational Design Synthesis (CDS) approach, as in [1] is applied. With reference to design issues, this CDS-centered software models can automatically provide design remedies and evaluate their relative performances. Thus, the users can effectively determine the spacing for the design remedies to assess promising designs, which create CDS-centered tools that apply the rule of the set-centered simultaneous engineering. Different software tools with the capacity to potentially automate the designs of production models have been assessed. Numerous approaches only recommend one solution for the design issues. In addition, most of these approaches handle the design parameters as the remedies of unbending design synthesis and search processes without providing any chances for designers to alter the generation of the design.

For it to possibly assure the benefits ignored by the present support and design automation approaches, this research paper indicates the implementation and rationale of the design support tools for the designing of the RCMS concept with reference to CDS. This tool helps individuals by automatically producing numerous design candidates so that individuals can iteratively evaluate and constrain the result spaces. The projected result is to reduce the time required for assessing the numerous designs of MS and to provide an intuitive method to evaluate the potentials in design and the effects of designs' decision on the performance of the system in a set-centered method.

This research is based on the fundamental concept: RCMS and CDS, which is a multidisciplinary scientific measure, which incorporates knowledge from different scientific disciplines e.g. knowledge engineering, computer science and constraint solving. In the mechanical engineering sector, the RCMS concept is utilized for the automation of the design analysis and synthesis of design candidates [2]. To enhance the process of making decision, CDS-centered software tools apply the algorithm procedures to formulate instantiation of the design framework. For this, the fundamental data about the requirements, structure, parameters and elements of the design remedies is based on the knowledge base, which incorporates the domain-based perspective, design processes, constraints and objectives.

Fig 1 below shows the computational support of the design actions. With reference to the descriptions, the result proposals are synthesized (path 1,2) and evaluated (3). Aftermath, the projected performances of the result proposal are assessed following two paths $(4,5)$. When the performance of the result proposal does not meet the specifications, designs are discarded and novel proposals are produced (6). As such, the remedy proposal becomes the candidate remedy 
$(7,8)$. Mechanical firms utilize CAx framework to underpin the evaluation of the remedy proposal as shown in the striped part in Fig 1. CDS-centered frameworks can extend the dimension of computational support of the wide-range activities of design evaluations and synthesis.

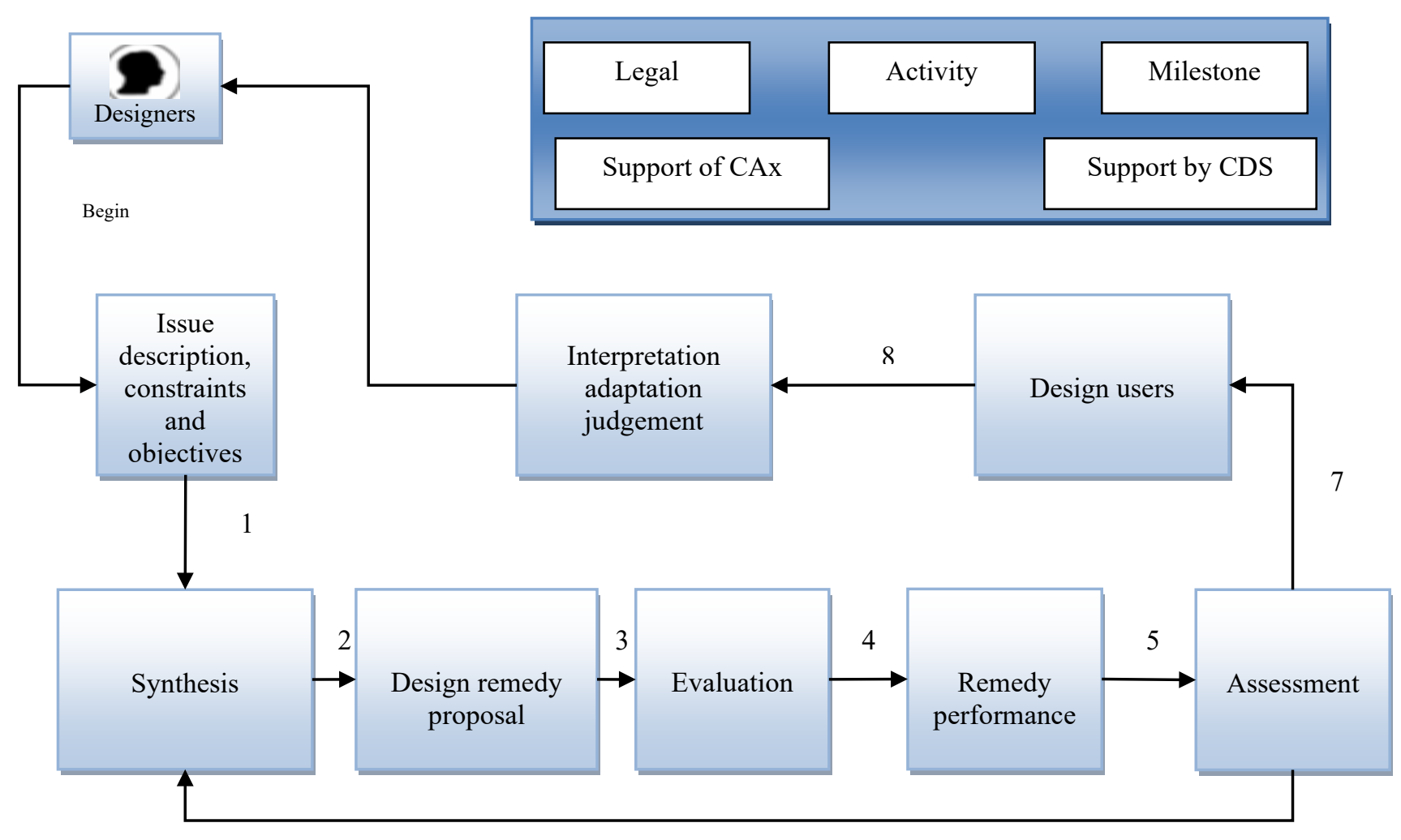

6

Fig 1: Computational support in the design activities

In case a high number of the potential design remedy can be produced, users can minimize the overall number by introducing constraints on the performance and design to produce remedies that meet the requirements. As such, the RCMS approach can be applied with the aim to explore the wide-range options based on the facilitation of remedy analysis and generation. Some of the various design segments whereby CDS has been applied effectively range from the design of gas supply networks to mechanical systems in engineering.

According to an analysis by P. Barash in [3], the designing of MS normally begins with conceptual design before the description of the design phases. In the conceptual design, the model architecture is structured and evaluated for its sustainability. Selecting a certain concept, i.e. RCMS, allows engineers to establish comprehensive designs for instantiating the wide-range design variables of RCMS. One of the most ignored activity in this stages, but significant, is the performance assessment of the wide-range design alternatives. The segment of operational research consists of mathematical methodologies for performance assessment, which incorporates the domain of MS.

According to our understanding, operational research is based on two various principles of supporting the design optimization, analysis and process as shown in Fig $2(\mathrm{a}, \mathrm{b})$ below. Based on assessment, it is comprehensive that mathematical models of MS are established with reference to the design candidates and based on the motivations to assess the performance of systems. Various levels of details can be references in the assessment; for example, by treating the performance features of the MS being stochastic and deterministic or for simulation of different cases.

Contrary to the assessment, optimization applies the algorithms, which can differentiate the system design parameters to identify the best performances based on the objective elements. An algorithm iteratively transforms the design parameters and evaluates the performances of novel designs until the remedy exhibits certain performances. Thus, optimization can be differentiated from the assessment, as the design synthesis steps are following using a computer. S. Vamvakidis in [4] have presented an analysis on the algorithms for the CMS design.

This paper has introduced the Computational Design Synthesis (CDS) approach, which is used for the automation of design tasks. The approach uses tools, which are used to mitigate design issues and assess relative performance. The 
approach uses the RCMS concepts to automate the analysis and synthesis of design candidates. The remaining parts of this part are organized as followed:

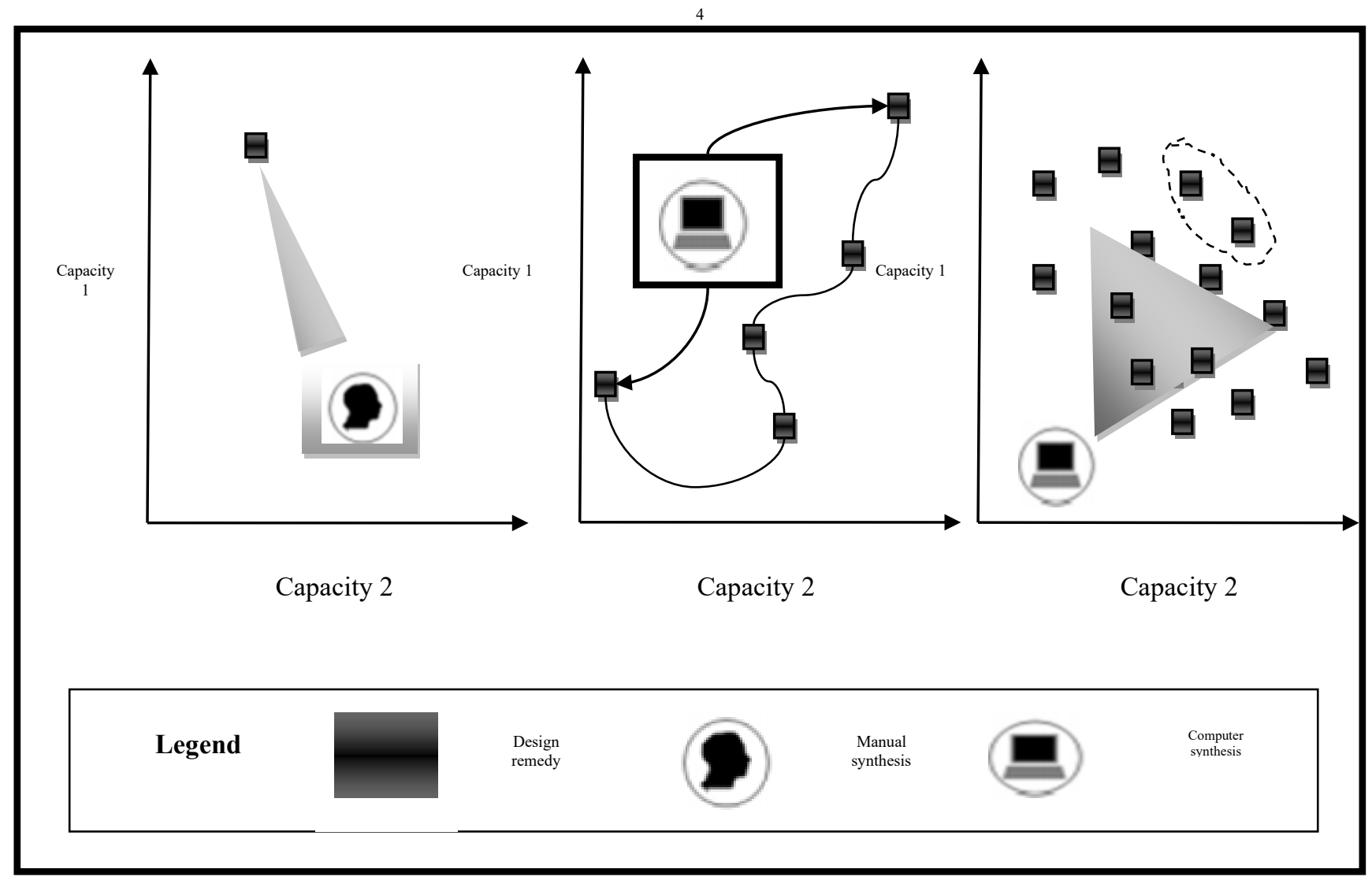

Fig 2: Design synthesis in optimization, analysis and CDS

\section{BACKGROUND ANALYSIS OF THE CONCEPT}

As seen in [5], the design of MS establishes Competitive Advantage (CA) for engineering firms. These systems unavoidably necessitate higher investments and signify the assets, which are depreciated over many decades. Therefore, decisions concerning the designs of MS have long-lasting effects and signify the commitments to the projected business developments. The business development is based on uncertainties can be challenging to focus. Based on the wide-range uncertainties, the operational research society and the engineering industry have created manufacturing concepts, which are based on the rapidly transforming demand for machine products.

The RCMS concept is one-category systems resulting from the operational research and it known to have particular characteristics as shown in Fig 3 below.

All the system elements are modular i.e. software and hardware to enhance changeability of the functional structures of the design. The integrality of the model component has to attain easy incorporation of the present and future manufacturing resources. Convertibility of the elements allows prompts changeover between the present-engineered products and allows adaptability of the design for the manufacturing of future engineered innovative products. System customization is meant to adjust the flexibility and capacities of the system to suit the present portfolio of engineered products.

According to H. Nouri and T. Hong in [6], RCMS provides a chance of mechanical engineering firms to establish productions, which are less exposed to uncertainties when contrasted to rigid MS. This is probably because these systems provide a chance for incrementally adapting and checking capacities and capabilities based on the marketing environment. Whereas the initial expenses of the system are assumed higher compared to the conventional designs, the application of RCMS focusses on the compensation by focusing on the combination of merits of dedicated MS i.e. affordable costs per segment with the flexible MS i.e. product mix flexibility.

RCMS shares the features i.e. modularity and objectives e.g. affordable costs per segment, which is through various ways. RCMS concentrates on attain manageable design of production and logistics process in the design system. For this aim, the same segments are segmented into segment families and production cells, which are established for every product family meant to attain low efforts necessary for operation and planning. In the production cell, robots are utilized to link up modules of the functional tools for manufacturing engineered products. 
Therefore, two vital concepts of RCMS and CMS are compatible. This resultantly maintains consistency of the present concepts; nonetheless, the terminology Reconfigurable Cellular Manufacturing Systems will be applied in the remaining sections of the paper. With the concept, modules of manufacturing cells represent personal production resources. Every module gives a particular manufacturing capacity to the cells, which are required for manufacturing, such as spot clinching and welding. Products are produced in more than a single cell dependent of the resource allocation throughout the cells in the design system. In case all the manufacturing phases of the engineered products can be performed on the functionality modules in a single cell, intermediate storage and transportation of the products is essential before processing can proceed to another cell.

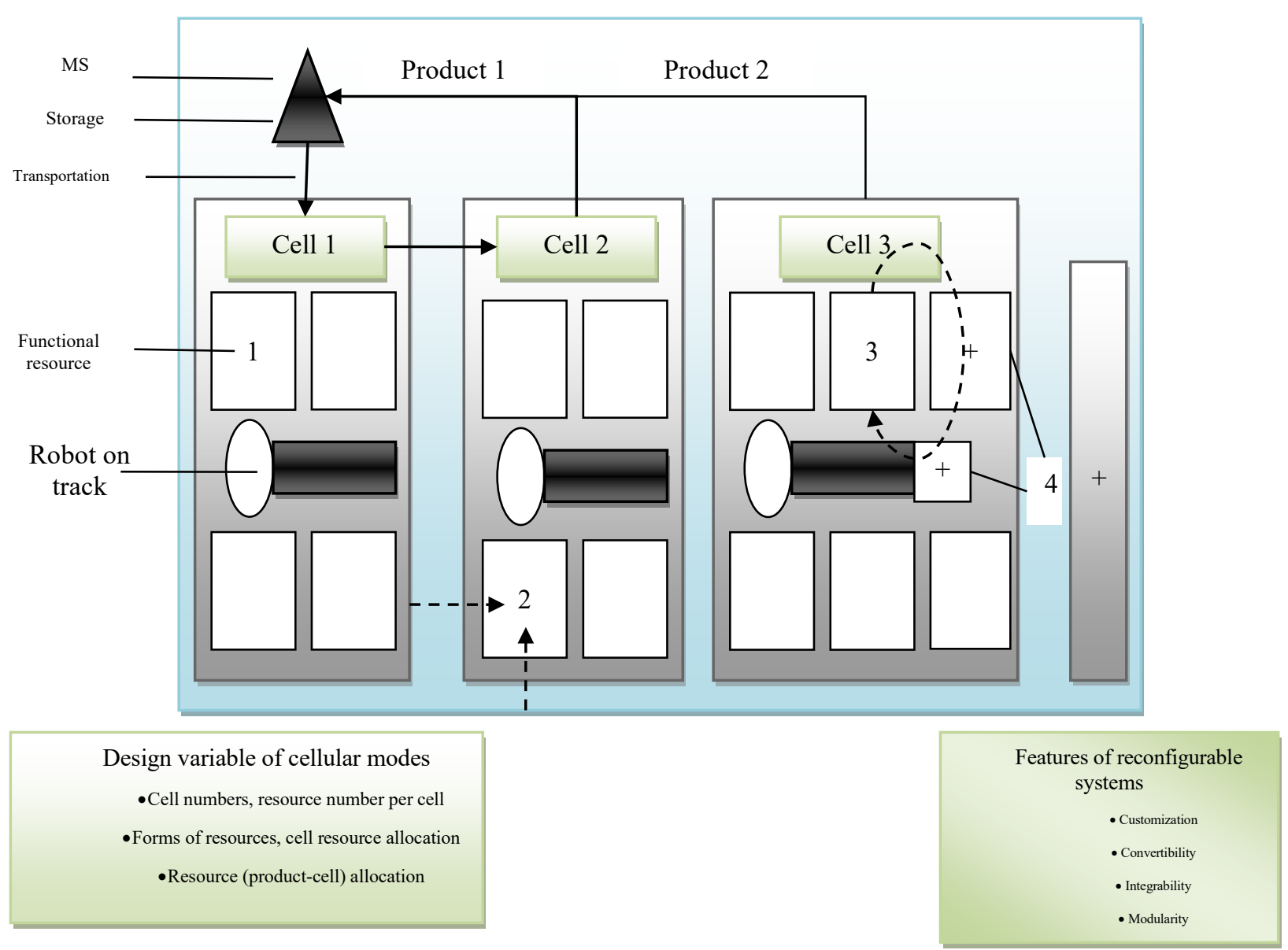

Fig 3: Features and design variables of RCMS

The development of the RCMS design is based on the capacities and capabilities of resources, which are allocated to a particular number of cells in the system. Based on the planning of the manufacturing products on a certain cell, links are created between cells in a manner of material flow. The order to the design phase (determining the cell number, allocating the manufacturing resources and allocating engineered products) is interchangeable and in every step, various decisions can be taken, therefore producing high numbers of designs that result to differing performance of the system.

According to assumptions by the operations research community, the issue is identified to as cell creation issue. Engineering firms and manufacturing models are complex social and technical engineered products from different corporate sectors and use distinct KPIs to assess the performance from varied perspectives (for cases from sales, logistics and finance). Even the transformations from a single system design variable can amount in different system behaviors and make performance assessments of different designs a time-intensive task.

Consequent to that, the adaptability and architecture of RCMS provides the designers of engineering system a widerange of chances to influence and design the performance of the system model that makes the model suitable for mitigating future uncertainties [7]. Nonetheless, high adaptability also establishes the opportunity of not utilizing the potential of manufacturing assets. To gain from the design freedom, the critical investigation of the effects of system design choices is required. As the transformations and reviews in the design happen frequently compared to the traditional schemes, the efficacy of design support is guaranteed. 


\section{LITERATURE REVIEW:}

T. Satake, S. Haramaki, M. Fujio and A. Hayashi in [8] argue that different software systems automating the design of MS have been defined for the segment of Flexible Manufacturing Systems (FMS). During the analysis, C. Saygin and S. Kilic in [9] created a professional design system, which has the capacity to automate the FMS design. To attain this, individuals are allowed to specify the performance obligations upfront to allow the system to project a single design.

S. Jovanović in [10] defines a system for the automatic design of FMS that can perform a wide-range design phases i.e. evaluating the workstations and allotting the tasks to workstations. R. Hicks in [11] illustrate the knowledge-centered tools, which are obliged to automatically produce FMS designs based on more than 1000 rules and assessments of performances. The research presents a prototype meant to support the FMS design that chooses the most effective control strategy and configuration for particular situations defined through an individual.

Y. Seo and P. Egbelu in [12] projected a support model, which assists in the designing of FMS by involving professional models with simulation modules and software for performance assessment in automatic loops. The analysis defines the flexible design automated model for assessing wide-range performances of different computer-aided assembly designs.

W. Gentleman, S. MacKay and D. Stewart in [13], over the past few decades assessed the different commercial software frameworks, which provide functionality for performance evaluation of MS such as Tecnomatrix and Anylogic, which are the approaches that can be viewed as sophisticated. It is fundamental to consider that the evaluation approaches are utilized based on the specified designs. This shows that the assessed design remedies are dependent on the concepts, which had been instantiated by the human decision makers. Resultantly, the design remedies are based on the constraints introduced during the instantiation of the design, which is a phase that influences certain design decisions that are unknown.

M. Khan, J. Butt, H. Mebrahtu, H. Shirvani and M. Alam in [14] attest that in optimization, designs are synthesized using computers. Nonetheless, the number and types of designs evaluated with optimization models have been limited using the previous models of the design, evaluating the algorithms utilized and relating the objective functions that determines the risks of not reflecting on the favorable design remedies. Moreover, the necessity to specify and assess the targeted value for performances in the objective element may be problematic. The objective of the assessment is to formulate initial overviews of feasible designs in distinct and various performance classes. Moreover, knowledge concerning the connections of design performance and decisions is normally based on mathematical models.

Z. Zhao, T. Wong, C. Leung and S. Probert in [15] have presented mathematical connection between the performance parameters and design to create and establish the model. In that case, numerous approaches are based on the experts in production modeling. However, other relevant stakeholders of MS with less knowledge on detailed relationships cannot apply this. The duration required for forming models and evaluating the performances plays a fundamental role, just like the duration of supporting the relative activities, and the constraints for designing MS to minimize the design proposals assessed. In that case, both optimization and analysis render the design procedures at risk of ignoring the design proposals, which are known for their satisfactory performances mostly in the aspect of wide-range design remedies.

R. Sykes and T. Travers in [16], argue that contemporary design support methodologies for MS based on performance assessment, but provide minimal guidance for actual designing activities and deficient options to create design assessment available to the interests of the relevant stakeholders with minimal modelling knowledge of MS. Even if some applications for the design automation of MS have been reported, they are known for their fundamental potentials that are unexplored. The researchers have also evaluated the methodologies for producing MS designs. Nonetheless, they produced a single design remedy for a particular issue, whereas the system signifies a methodology that covers a wide-range design phases, consideration is minimized to one design remedy.

A. Kamrani, H. Weibe and C. Wei in [17] have assessed a single design automation model among the wide-range tools earlier presented to product multiple remedies and assess their performances in a comparative manner. In the support framework, the remedies are either synthesized or optimized based on smart algorithms or under comprehensive analysis. A possible approach to the issue of search space evaluation, which has not been defined in the reported system, nonetheless, is the role, which users can play in evaluating the solution spaces. The rationale identified in this case in most systems is that the algorithms are utilized for choosing, analyzing and synthesizing the design proposal with reference to rigid processes, which leave users with minimal opportunities to alter the functionality of the design parameters of the remedies.

J. Davila Delgado and H. Hofmeyer in [18] projected that the automation of designs can be utilized in facilitating the accessibility of remedy spaces based on the application of experiences and knowledge for choosing suitable designs. The capacity of automatic design is applied in the proposal of multiple feasible remedies to the users hence permitting the evaluation of the remedy spaces, specifying (performance and design) constraints and hence iteratively minimizing the remedy spaces to manageable sizes. No such methodology has been assessed. The application features of RCMS makes the system form a viable chance to evaluate the advantages and disadvantages of supporting MS design in this manner. Ultimately, the objective is to facilitate the set-centered design decisions on the configuration of the systems.

\section{CRITICAL ANALYSIS}

This chapter describes the RCMS concept that has been implemented in the prototype software framework and the potential advantages in the context of the design procedures. 


\section{A. CDS-Centered Support Scheme for Designing RCMS Concept}

In this part, an approach has been projected with an objective of providing more benefits from the design automations compared to the accomplished design support models. To effectively uncouple the manifestations of modeling knowledge from the design production, we have projected a knowledge-centered scheme. Our state-of-the-art prototype software supports the design with the aid of CDS (Fig 4).

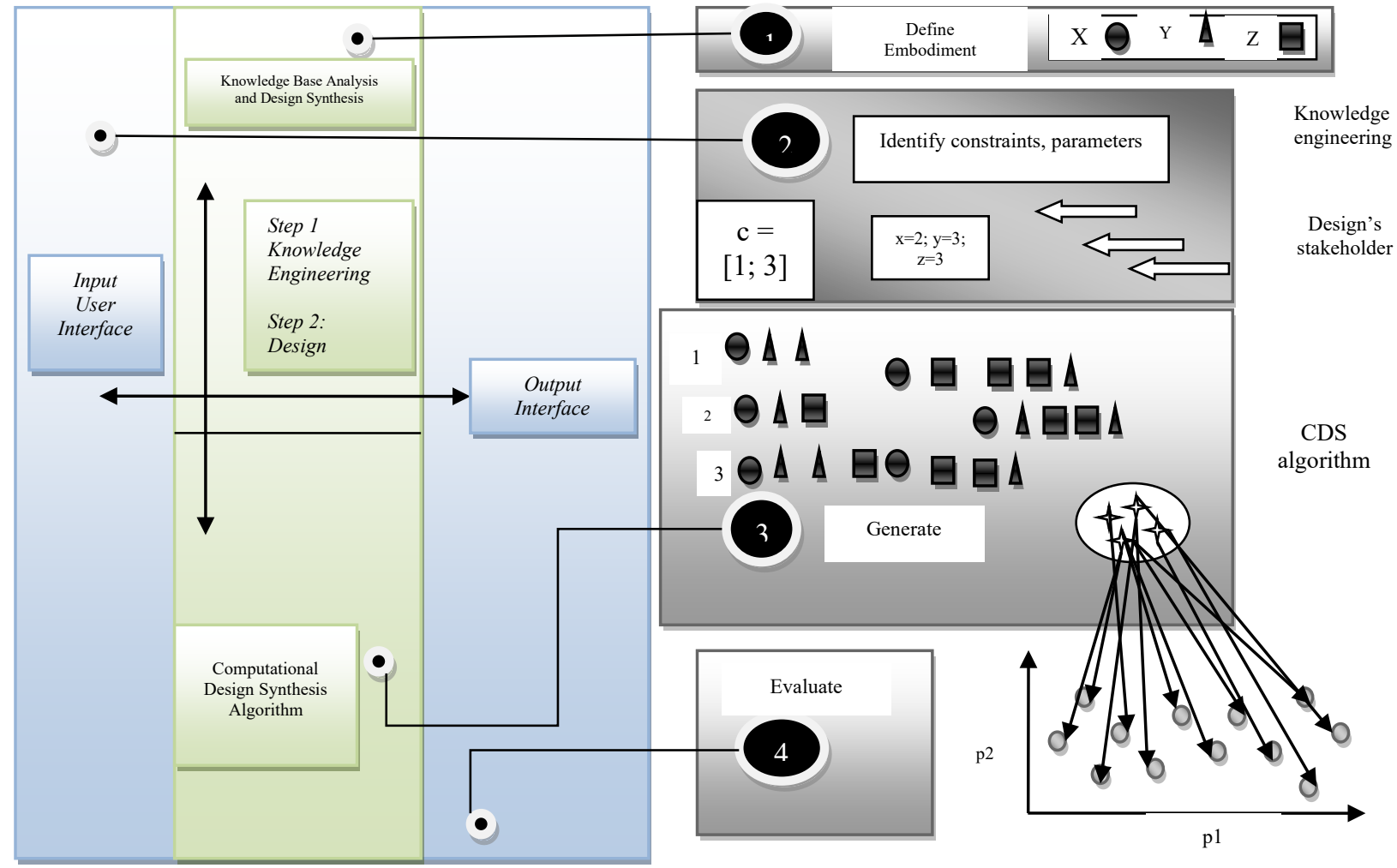

Fig 4: CDS-centered support model and concept for RCMS design

\section{1) Structure of the CDS-Centered Support Scheme}

Before defining the components of the support scheme, the context of application with two fundamental activities: design instantiation and knowledge engineering have to be evaluated.

\section{a) Step 1 Knowledge Engineering}

The purpose of knowledge engineering is to effectively capture professional knowledge about the overall logical and parametric relationships in domain specific design analysis and synthesis. To store this data, the design knowledge base is formed in the software, which gives the domain specific definitions of the design embodiments. As for RCMS, knowledge base incorporates the descriptive data concerning the design objects and their relative performances, such as the production cells [19]. The entire system includes the manufacturing resources, which create the cells and the logical connections between cells, systems and resources. This overall model has to be compatible to apply CDS algorithms to resolve dependencies and relations.

\section{b) Step 2: Design Instantiation}

When a complete knowledge framework is available, the users provide input data to instantiate the design remedies, which match a particular context of the design issue. The input for this tool can be differentiated into two various classes: solution requirements, solution components and scenarios. Scenarios can be utilized to illustrate the uncertainties of the system environment whereby the other two inputs illustrate the requirements and opportunities for the system design.

The way and format of expressing this data is based on the connections are defined in the knowledge base. Inputs are utilized for computational analysis and synthesis of the design remedies. Aftermath, the results are then presented to users to signify the tool output, design remedies and their performances. Whereas the features of the design issues, which reflects on the issue of basic representation, analysis and dependency steps do not transform. As a result, the same knowledge base can be applied to produce the design remedies for different parametrization of cases, requirements and solution elements.

\section{2) Elements of CDS-Centered Support Scheme when Applied to RCMS}

a) .Knowledge Base Analysis and Design Synthesis

Knowledge base incorporates descriptive data concerning the relations and structure of RCMS objects. In knowledge base, the representation of the MS is based on the levels of cell modules, which incorporates the system and cell levels as 
seen Fig 4. With this, the context between the general system design and the separate design components are formed. Moreover, the analysis approaches utilized to evaluate the performances of the framework are incorporated in the knowledge base, including the fundamental representation of cases to consider various system environments during analysis.

\section{b) Input User Interface}

The input user interfaces acts for configuration of the wide-range design synthesis sessions whereby the users specify the parameters for the case remedy components and the remedy requirements. Uncertainties can be attained based on the formulation cases, for example, quantification of various expectations of upcoming product demand based on the manufacturing resource requirements, which can be utilized for design analysis. In that case, the interface gives features, which aid the users in producing multiple settings of design remedies. These remedies are exposed after the synthesis process and aid in the process of attaining differential system impression of particular discrete designs.

Moreover, the interface is utilized to specify discrete values of the parameters of the design components, for example, in the process of assigning the processing and investment speed for every module of machine utilized in the system design. In addition, the performance requirements and the design constraints for the design remedies are formulated in the component (see phase 2). In the RCMS design, the constraints can therefore be imposed, for instance, on the overall system investment or the overall cell number of the system design.

\section{c) Computational Design Synthesis Algorithm}

When the concrete cases, the values of the relevant features of the remedy components and requirements, are present, the design is instantiated. For this aim, the design remedies are therefore synthesized by the algorithm process and based on the protocols specified in the knowledge base. An illustration of routine application in our design prototype of RCMS is provided in phase 3 . The system design can be evaluated for every product case to evaluate the performance design (phase 4). Aftermath, validity of the design remedies are tested through the comparison established on the design and the performances of the constraints of users.

\section{d) Output Interface}

In the final step of utilizing tools, design remedies and their performances are visualized when they satisfy the specifications of users. The interfaces support the users in the process of comparing and exploring the potential designs (phase 5). Users are allowed to evaluate the influence of various cases, which allow the users to identify the most suitable remedy and provide indication to ease and impose constraints on the system embodiment and performance. The current structure allows the specification design requirements, provide multiple design remedies and minimize the number of the design remedies considered through the advent of higher necessities of remedies in an iterative manner. After selecting the promising design, the design suitability can be assessed and validated with traditional support methods for MS design.

\section{Key Design Parameters and Synthesis Process}

The key design parameters utilized to model the system and to synthesis, procedures are dependent on particular context of application. In our assessment and implementation, we utilize the parametric design synthesis, whereby the key design parameter include the system cell, number of manufacturing resources allocated to the cells and the assignment of engineered products to particular cells and resources. Consequent to that, the combination number potential to synthesis is based on the values that can assign the constraints and parameters of users. Since the issue of cell creation is a combinatorial one, we utilize the heuristics, which assess the manufacturing requirements of the manufacturing resources.

The boundaries are determined for different design strategies that the individuals select. For instances, individuals can select a strategy design to obtain systems whereby every product family is completely produced in its individual manufacturing cell. Another assessment strategy is to produce system designs, where all the engineered products use a single route over multiple cells. For every design remedy to be produced, random algorithms will instantiate the value in the permitted dimension of the design parameter to retrieve a completely specified design.

The final is the heuristics-centered assignment of engineered products to the system resources. For every resolved design parameters, the constraint-solving algorithm determines where the constraints have been satisfied or not. In case the limit value for these parameters have been misused, for example by surpassing the limitation of the general investment when incorporating additional machines to the manufacturing cells, the design is typically discarded and a novel one is formed. In this way, individuals indicate the design approaches, which seem interesting to them and potentially specify the alternative remedies to be produced. Therefore, algorithms attempt to produce this number in a particular timeframe. In case no remedies can be provided, the constraints are relaxed.

\section{B. Projected Effects}

The higher numbers of various decisions, which can be taken when formulating RCMS, can make the effect on the performance of the system challenging to assess. The practical applications of the common decision support methodology, e.g. evaluation, can show that the makers of decisions have no constrain count of design alternatives due to time constraints. Resultantly, this amounts to the exclusion of valuable remedies from being considered. Applying the current CDS-centered tools before the application of traditional methodologies for assessment of detailed system designs has to aid 
in uncoupling of the range of remedies assessed using traditional methods [20]. Based on this, unwanted effects through the application of the tools will compare and generate multiple and feasible designs without necessitating the designing of explicitly specification of design remedies or their advance objectives.

Moreover, knowledge base has to allow reuse of the present knowledge and allow for the generation of more feasible designs without requiring the knowledge of owners to be available at that moment of design assessment and generation. Through front-loading of the system design procedure, tools have to aid in shortening the duration required for producing and assessing the design alternatives that are certainly fundamental for the recurrent design activities in RCMS.

Another projected effect is to develop the capacity to assess and generate the design to more individuals than the experts themselves and to utilize the tools in facilitating discussions and evaluations of the design effects. In this measure, the relevant stakeholders have to focus on the capture of perceptions of the uncertainties of the cases and select the most performing designs definitely. The stakeholders are allowed to develop the experimental attitude to MS and to get the indications of the sensitivity of the design to various cases. With this, the current tools provide a means of allowing the setbase concurrent engineering and show the available opportunities irrespective of the conflicting performance objectives to enhance the design quality based on the lobbyists of various corporate functions.

\section{Assessments}

Critical assessment has to be undertaken to assess the application of the tools based on the current design issue. The engineering firm facing the issue of RCMS design in the high-to-mix and low-to-volume manufacturing of spare parts in the mechanical engineering firm stimulates the structuring of prototype. In that regard, the objective is to assess the effects of applying software in the industrial case. For this context, the Design Research Methodology was implemented.

The development stage was complete through the development of the software prototype of design automation framework. The major objective of this stage was to generate a system, which fulfills the requirement of industrial users in terms of interaction tools, data output and input. For this aim, the elicitation of workshop knowledge was held in the engineering firm to assess the performance of the system utilized in the evaluation of designs, production systems and resource representation, including the analytical dependencies between the performance and design.

Another workshop concentrates on the case formulation and allowing the detection the basic parameters, which render potential use situations of MS in the form of engineered product cases. Moreover, an assessment was done to identify suitable user-tool interaction mechanisms in the design assessment and generation based on lo fidelity prototypes. Based on the results of the activities, the development stage concluded with the formulation of knowledge base, which can be utilized in the combinations with randomize algorithms and constraints solver to automatically analyze and design the system configuration. To generate data input and output, suitable interfaces of the users are created.

The evaluation stage will be the next phase. In this stage, the experiments will be done based on validation and verification of the enhanced approach. The verification research has to make user that the prototype is able of analyzing and synthesizing designs of MS and presenting them in reference to the specifications of the users. Interesting aspects to be perceived in this stage will be, if the assessed analytical models will permit the users to detect fundamental alterations between designs and when these variations can be interpreted. Moreover, the model sensitivity and model remedies to constraints will be assessed.

Lastly, an empirical research will be accompanied to assess if the projected effects of the design support methodology can be authenticated. Interesting query in this context has to reveal the advantages of utilizing the tools, such as: Does the application of the support yield various designs compared to the traditional synthesis? How does it influence the design enhanced? Does the support aid in the speed up the result generation? Does the support aid the makers of decisions in producing a comprehension of the design issue? Does the option to incorporate the stakeholders into the design facilitate or complicate the design process, if they have direct or indirect effect on the design. Moreover, to assess the current design support, we anticipate to find out about the fundamental aspects of industrial and acceptance application of decision support in MS design and to get fundamental insights for more research into the automation of design and set-based designs for MS.

\section{CONCLUSION AND FUTURE DIRECTIONS}

In conclusion, this research contribution evaluates the significance of MS for engineering firms and users within. It evaluates that when manufacturing firms are exposed to production concepts and demand uncertainties such as RCMS can be applicable strategy. Nonetheless, the higher number of different system performance and structure makes it essential to support the design choices. Common decision support approaches utilized for the design of MS are defined. In addition, the present support systems based on design automations are explained and a new purpose is noted for which the automation of the design can be utilized. With reference on this, the major chapter indicates the rational and structure of implemented CDS-centered software tools, which has to aid the users to derive more usage out of the possible automation of design.

Consequent to that, mechanical engineering users could anticipate such tools to support the design procedures. Most significantly, the tool should allow individuals to concentrate on the assessment of the solution spaces and formulating designs and performance-based constraints. The future tool should be used as a design support that evaluates the wide- 
range design remedy compared to the present approaches. Ultimately, the mechanical engineering context of development of the prototype tools should be evaluated and planned in the future empirical research for the tools' assessment.

\section{References}

[1]. M. Campbell and K. Shea, "Computational design synthesis", Artificial Intelligence for Engineering Design, Analysis and Manufacturing, vol. 28, no. 3, pp. 207-208, 2014. Available: $10.1017 / \mathrm{s} 0890060414000171$.

[2]. M. Raza and R. Harrison, "Information Modeling and Knowledge Management Approach to Reconfiguring Manufacturing Enterprises", SSRN Electronic Journal, 2011. Available: 10.2139/ssrn.3424778.

[3]. P. Barash, "Artificial intelligence and expert systems in manufacturing: The scope, applications, and limitations of intelligent manufacturing systems", Journal of Manufacturing Systems, vol. 10, no. 4, pp. 350-352, 1991. Available: 10.1016/0278-6125(91)90030-6.

[4]. S. Vamvakidis, "Computational Design Thinking through Controlled Transformations: An Analog Computational Design Approach", The International Journal of Design Education, vol. 15, no. 1, pp. 177-191, 2021. Available: 10.18848/2325-128x/cgp/v15i01/177-191.

[5]. K. Tupamahu, I. Ghozali and P. Basuki, "Lean Management, Competitive Advantage, and Firm Performance: The Role of Management Control Systems (Evidence from Indonesia Manufacturing Firms)", Academic Journal of Interdisciplinary Studies, vol. 8, no. 3, 2019. Available: 10.36941/ajis-2019-0020.

[6]. H. Nouri and T. Hong, "Development of bacteria foraging optimization algorithm for cell formation in cellular manufacturing system considering cell load variations", Journal of Manufacturing Systems, vol. 32, no. 1, pp. 20-31, 2013. Available: 10.1016/j.jmsy.2012.07.014.

[7]. M. Ali, A. Baqai, S. Butt and S. Hasan, "Framework for Part Families Associated with Setup Sequence Based Similarity in Reconfigurable Manufacturing System / Cellular Manufacturing System", International Journal of Materials, Mechanics and Manufacturing, vol. 6, no. 1, pp. 6368, 2018. Available: 10.18178/ijmmm.2018.6.1.348

[8]. T. SATAKE, S. HARAMAKI, M. FUJIO and A. HAYASHI, "Development of Software-Toolkit for Reconfigurable Software Applications for Manufacturing Systems(Advanced Manufacturing,Session: MP2-D)", The Abstracts of the international conference on advanced mechatronics : toward evolutionary fusion of IT and mechatronics : ICAM, vol. 20044, no. 0, p. 36, 2004. Available: 10.1299/jsmeicam.2004.4.36 3.

[9]. C. Saygin and S. Kilic, "Dissimilarity Maximization Method for Real-time Routing of Parts in Random Flexible Manufacturing Systems", International Journal of Flexible Manufacturing Systems, vol. 16, no. 2, pp. 169-182, 2004. Available: 10.1023/b:flex.0000044839.27404.92.

[10]. S. Jovanović, "Flexible Manufacturing Systems and Quantitative Anlysis of Flexible Manufacturing Systems", International Journal of Computer Applications, vol. 132, no. 1, pp. 6-14, 2015. Available: 10.5120/ijca2015907243.

[11]. R. Hicks, "Knowledge base management systems-tools for creating verified intelligent systems", Knowledge-Based Systems, vol. 16, no. 3, pp. 165-171, 2003. Available: 10.1016/s0950-7051(02)00082-5.

[12]. Y. SEO and P. EGBELU, "Flexible guidepath design for automated guided vehicle systems", International Journal of Production Research, vol. 33, no. 4, pp. 1135-1156, 1995. Available: 10.1080/00207549508930197.

[13]. W. Gentleman, S. MacKay and D. Stewart, "Commercial realtime software needs different configuation management", ACM SIGSOFT Software Engineering Notes, vol. 14, no. 7, pp. 152-161, 1989. Available: 10.1145/73337.73365.

[14]. M. Khan, J. Butt, H. Mebrahtu, H. Shirvani and M. Alam, "Data-Driven Process Reengineering and Optimization Using a Simulation and Verification Technique", Designs, vol. 2, no. 4, p. 42, 2018. Available: 10.3390/designs2040042.

[15]. Z. Zhao, T. Wong, C. Leung and S. Probert, "Wok design: thermal-performance influencing parameters", Applied Energy, vol. 83, no. 4, pp. 387400, 2006. Available: 10.1016/j.apenergy.2005.04.002.

[16]. R. Sykes and T. Travers, "Dossier: Contemporary Methodologies", Dandelion: Postgraduate Arts Journal and Research Network, vol. 8, no. 1, 2017. Available: $10.16995 / \mathrm{ddl} .367$.

[17]. A. Kamrani, H. Weibe and C. Wei, "A Stochastic Model for the Analysis of Robots Performance", Journal of Design and Manufacturing Automation, vol. 1, no. 3, pp. 175-191, 2001. Available: 10.1080/15320370108500208.

[18]. J. Davila Delgado and H. Hofmeyer, "Automated generation of structural solutions based on spatial designs", Automation in Construction, vol. 35, pp. 528-541, 2013. Available: 10.1016/j.autcon.2013.06.008.

[19]. D. WANG, Y. HUANG and X. CHEN, "Design and implementation of geometric knowledge base", Journal of Computer Applications, vol. 29, no. 2, pp. 398-402, 2009. Available: 10.3724/sp.j.1087.2009.00398.

[20]. M. Wenger et al., "CDS Tools for Cross-Identification", Open Astronomy, vol. 9, no. 4, 2000. Available: 10.1515/astro-2000-0427. 